\title{
Quaderni
}

QUADERNI Communication, technologies, pouvoir

89 | Hiver 2015-2016

Penser l'avenir : le CESTA, un think tank atypique

\section{Un récit métropolitain en construction au Perreux-sur-Marne}

Bertrand Morvan

\section{CpenEdition}

Édition électronique

URL : http://journals.openedition.org/quaderni/961

DOI : 10.4000/quaderni.961

ISSN : 2105-2956

Éditeur

Les éditions de la Maison des sciences de l'Homme

Édition imprimée

Date de publication : 5 janvier 2016

Pagination : 87-101

Référence électronique

Bertrand Morvan, «Un récit métropolitain en construction au Perreux-sur-Marne », Quaderni [En ligne], 89 | Hiver 2015-2016, mis en ligne le 05 janvier 2018, consulté le 19 avril 2019. URL : http://

journals.openedition.org/quaderni/961; DOI : 10.4000/quaderni.961

Tous droits réservés 


\section{Un récit métropolitain en construction au \\ Perreux-sur-Marne}

\section{Bertrand Morvan}

\section{Conseil en développement territorial \\ Docteur en science politique Université Paris Panthéon-Sorbonne}

Le Perreux-sur-Marne, commune de 30248 habitants de la banlieue Est de la région parisienne, a commencé, fin janvier 2015, une phase de concertation avec les habitants pour définir le nouveau plan local d'urbanisme (P.L.U.). Elle occupera plus d'une année de concertation. Une première phase préparatoire du P.A.D.D. (plan d'aménagement et de développement durable) suit le diagnostic territorial. En juillet 2014, 19 contrats territoriaux de développement illustrent le projet métropolitain. Ces contrats représentent des outils d'un processus d'institutionnalisation du local d'un «État-animateur » (P. Estèbe, J. Donzelot, 1994). Ils accompagneront de futurs redécoupages et de nouvelles intercommunalités en limite de première couronne parisienne. Les communes de cette périphérie devront en être parties prenantes, à l'horizon 2016, en parallèle d'une redéfinition de l'espace communal à l'aide de leur P.L.U.

Avec le projet de Grand Paris consacré par la loi du 27 janvier 2014 sur la modernisation de l'action publique territoriale et l'affirmation des métropoles, une controverse est née entre élus du rural et de l'urbain (en province) et entre élus du périurbain et de la ville-centre (sur la métropole parisienne). La loi « N.O.T.R.e. » (Nouvelle Organisation Territoriale de la République) l'a illustré dès la fin de l'année 2014. Avec l'intervention du Sénat en seconde lecture et un retour du projet de loi pour un vote à l'Assemblée Nationale, la loi sera « retoquée ». Un report de l'application est prévu pour janvier 2016. Une nouvelle répartition des compétences accompagnera la création d'une métropole au statut particulier. Une négociation conflictuelle va organiser le rapport entre local et global, entre 
élus régionaux et municipaux et entre Sénat et Assemblée Nationale sur le périmètre attendu et la mise en application.

Par ailleurs, des spécialistes de la question urbaine (P. Veltz, L. Davezies, 2014, 2015) plutôt économistes, ont pris acte des inégalités territoriales accentuées par les mutations urbaines. Une « interconnexion généralisée » $(\mathrm{O}$. Coutard, G. Dupuy, 2013, 2014) devient de plus en plus chère et inefficace avec ses développements récents pour la métropole parisienne. Il faudrait noter par exemple, la dépendance créée et le manque de souplesse des projets d'infrastructures (G. Dupuy, 2013). On peut, de moins en moins, compenser les effets déséquilibrants de formes urbaines métropolitaines prédéterminées ${ }^{1}$.

Le passage d'une question sociale à « la nouvelle question territoriale » (L. Davezies, T. Pech, 2014) oblige à reformuler un contrat social à partir des questions de cohésion territoriale et de solidarité républicaine. Ces ingrédients sont de plus en plus incertains à trouver. Dynamiques économiques et inégalités sociales croissent de façon exponentielle et sans corrélation avec le modèle dominant de développement local. Le développement de territoires insuffisamment spécialisés est laissé aux mains de la puissance publique. L'intervention des acteurs économiques dominants des secteurs de l'économie avancée pourrait se révéler être un handicap. Une lecture nationale et infra-métropolitaine des dynamiques urbaines l'illustrerait avec l'hypothèse générale d'une « économie en archipel» (P. Veltz, 1996). De quoi s'agit-il ? Avec ce processus de mondialisation économique, des territoires de plus en plus spécialisés et interconnectés émergent comme des îlots formant archipels. Le reste des territoires se fractionne. Ils sont de plus en plus ségrégés à partir de formes urbaines héritées de la période industrielle. Elles vivent des ségrégations socio-spatiales qui s'accentuent par rapport aux centralités existantes. Des dynamiques métropolitaines sont en partie observées, et attendues, à l'aide des modèles de croissance urbaine qui s'opposent parfois. Seuls des projets de territoires, les plus attractifs possibles et soutenus par les pouvoirs publics, devront relever le défi de points aveugles « d'une nébuleuse de points émergeant de façon multipolaire et composant un polycentrisme hiérarchisé »(P. Panerai, 2008). L'institutionnalisation du local métropolitain, dès juillet 2014, avec des contrats de développement territoriaux (C.D.T.), va tenter ce rééquilibrage des perspectives.

Pourtant, certains travaux des équipes de l'Atelier International du Grand Paris poseraient le problème d'une telle interrogation, mais de manière sibylline et indirectement avec le sens que prennent les stratégies de projets urbains proposées. Il s'agirait, en aval, d'interventions proposées sur la forme urbaine existante pour construire de nouvelles centralités à forte symbolicité en banlieue ${ }^{2}$.

Un constat général de désengagement de l'État sur le plan économique, technique et financier est pourtant noté aujourd'hui sur la région urbaine, à la différence de l'amorçage par les pouvoirs publics du projet de Grand Paris en 2008, selon F. Léonhardt, consultant spécialiste de politiques de renouvellement urbain à l'agence nationale de rénovation urbaine (A.N.R.U.) lors d'une intervention le 14.04.2015 à l'A.C.A.D., association de consultants en développement territorial. La 
seule exception reste alors le travail d'animation stratégique avec les 19 C.D.T. (contrats de développement territoriaux).

Nous nous proposons d'explorer l'hypothèse d'un « local métropolitain » résistant à la métropolisation (selon l'introduction du géographe D. Béhar lors de la $6^{\mathrm{e}}$ session du séminaire du Grand Paris, E.U.P., 19-20.03.2015) et la question des conditions d'une participation démocratique. Les réunions publiques apparaissent comme des dispositifs de consultation des populations dans les communes de la première couronne parisienne attirant une population en nombre. Sont-elles représentatives de l'ensemble des perruxiens lors de conférences-débats ?

Il s'agirait alors de réinterroger la capacité des citoyens et des pouvoirs à être interpellés et à se positionner, démocratiquement, par exemple, dans une forme alternative avec des ateliers d'urbanisme pédagogiques. Il s'agit là d'une proposition que j'ai faite mais qui n'a pas été retenue par l'édile du Perreux, car venue trop tard et contre-productive, selon lui, par rapport au processus en cours (réponse datée du 30.01.2015 des services techniques et du cabinet du député maire). L'évaluation de l'efficacité symbolique d'une « fiction (ou récit) techno-politique » (L. Sfez, 2002) de l'édile, composée de "métaphores vives » (P. Ricœur, 1975, 1985), de « représentations sociales » (S. Moscovici, 1969, 2002) ou encore de « croyances et représentations idéologiques » (J.F. Deconchy, 1981) s’impose alors.

Deux orientations d'enquêtes seront engagées sur le Perreux-sur-Marne en partant de deux types de sources. Il s'agit d'une part, de l'observation des réunions publiques organisées par la Mairie du Perreux et le cabinet-conseil Citadia (sa filiale «Aire urbaine ») avec le député maire. L'objectif en est la définition des grands axes de développement du P.A.D.D., les avis des habitants déposés sur le site internet de la ville et ceux figurant sur un recueil à l'entrée de la mairie. D'autre part, et pour l'essentiel, nous nous intéresserons aux éléments de récit de l'édile. Il faut l'appréhender comme une médiation symbolique « charnière » entre toutes les composantes mentionnées précédemment. Il se constitue en effet, au contact des acteurs et habitants avec une dimensions historique forte, constamment mise en avant comme « repère » pour les habitants.

\section{Les enjeux du projet de ville du Perreux selon la programmation politique et urbaine jusqu'à l'horizon 2016}

Les phases de la concertation au Perreux-surMarne peuvent être présentées de la façon suivante :

1. le diagnostic (où en est le territoire ?);

2. le projet (P.A.D.D.) en juin 2014 ;

3. les règles : Règlement et zonage avec les réunions publiques (sur 3 mois) pour dégager les axes du zonage présentés en juillet-octobre 2015, avec l'arrêt de la concertation ;

4. la procédure de validation en avril-juin 2016, puis l'application.

Lors des réunions publiques du 28 janvier et 6 février 2015, deux questions sont soumises avec tout d'abord : les P.L.U. doivent-ils rester communaux ou passer à l'échelon de la métropole 
régionale avec le débat au sein de la commission du Parlement? Et ensuite : comment répondre à l'objectif de l'État (création de logements sociaux), sous le contrôle de l'État?

Selon le député maire Gilles Carrez, porteur principal du projet de renouvellement du P.L.U. avec l'accord de la majorité municipale en décembre 2013 : «Le P.A.D.D. doit être envisagé à partir des espaces constructibles. Il faut ensuite décider quels types d'équipements choisir et quels sites protéger (espaces verts, etc.). Cela engage sur 10 ou 15 années l'avenir de la commune. Le diagnostic initial porte sur la démographie, l'habitat et les équipements existants, permettant ensuite une projection à partir d'estimations pour l'avenir... à prévoir pour 20 ou 30 années. On peut déjà escompter un accord sur les schémas supra-communaux d'organisation de l'espace, P.A.D.D. et S.C.O.T.(schéma de cohérence territoriale intercommunal remplaçant l'ancien schéma directeur). Le P.L.U. lui, mettra plus de temps à être élaboré ».

En ce qui concerne la gouvernance institutionnelle, «s'il y a intercommunalité nécessaire des perspectives pour répondre aux termes du contrat de développement territorial signé avec l'État, chacun des maires devrait rester autonome quant à son pouvoir administratif (il s'agit ici du contrat de développement territorial dit 'des Boucles de la Marne')». Préserver son pouvoir d'urbanisme communal et défendre son mandat est présenté comme une nécessité face aux mises en cause de la loi A.L.U.R. et des objectifs de création de logements imposés par les pouvoirs publics.

Le cabinet d'urbanisme Citadia-conseil, mis- sionné par le député maire, a pour premier enjeu de la commande, la préservation du cadre de vie et ce qu'il nomme « trame verte et bleue». Il s'agit des paysages à préserver : parc, centre historique, quartier des Joncs marins, bords de Marne, mais aussi points de vue, maillage des bandes cyclables, entrées de villes à requalifier, et patrimoine bâti avec plusieurs types de maisons : maisons de maîtres, maisons éclectiques...

Le deuxième enjeu concerne la dynamique urbaine. La population croît depuis les années 80. Le nombre de personnes âgées et de familles nombreuses augmente. Il faut donc plus de logements (355 logements sociaux par an dans le contrat de développement territorial signé avec l'État). Par ailleurs, le volet « emplois », surtout tertiaires, est concerné. Des objectifs stratégiques et structurants doivent être renforcés : centre-ville, gare Neuilly-Plaisance, le boulevard Alsace-Lorraine (R.D.34). Il faudrait développer les relations avec Fontenay-sous-Bois et la venue du Grand Paris Express.

Pour le cabinet : « Face à la saturation des axes routiers, des contraintes environnementales et l'usage de modes doux de locomotion sont à envisager. Il faut anticiper des impacts environnementaux (eau, assainissement, problèmes de risques et nuisances; plan de risque des mouvements des sols; sonorité). En ce qui concerne l'occupation du sol : 3/4 de la superficie communale est dédiée à l'habitat. Des espaces naturels sont à préserver. Un seul secteur est déjà identifié pour le futur développement: le cour de ville, dans le secteur de la gare de Nogent-Le Perreux, l'avenue Brossolette et le R.D.34 (335 logements/an à créer pour la communauté d'agglomération) ». 
Qui est Citadia-conseil et comment décrire la stratégie d'action de ce bureau d'étude ? Sa filiale «Aire publique », agence de communication institutionnelle, a pour mission «l'accompagnement des acteurs du territoire dans leurs projets d'aménagement et de développement » (cf. Présentation d'Aire Publique, filiale de Citadia-conseil : « Communication et concertation », en ligne, pp. 6 à 10, 2015). Elle le fait en particulier, pour les politiques contractuelles dont le plan local d'urbanisme. Elle s'est constituée en 2003 pour l'association de compétences complémentaires : communication, marketing, graphisme, urbanisme et développement local pour " garantir aux porteurs de projets un conseil pragmatique et efficace associé à une parfaite compréhension des enjeux territoriaux et stratégiques de chaque mission ».

Pour la concertation : «Une assistance globale des décideurs locaux propose la prise en charge complète des procédures de concertation : étude de contexte, définition de la stratégie, identification des acteurs, sélection des outils de mobilisation et création de supports d'information, prise en charge du matériel, animation de rencontres et suivi de la démarche de participation ». «En favorisant l'échange et le dialogue, les démarches de concertation mises en place permettent de sensibiliser et de faciliter l'appropriation du projet par l'ensemble des acteurs : élus et techniciens, société civile, acteurs économiques, habitants... » Le pilotage des procédures de concertation passe par des réunions publiques, ateliers participatifs, forums, tables-rondes, marches commentées, jeux de rôle. En ce qui concerne l'élaboration et l'analyse de processus de consultation : « il s'agit d'enquêtes de terrain, questionnaire administrés, entretiens stratégiques, sondages qualitatifs...». Des journées de formation sont conçues pour un public non averti ou techniciens. Aire publique « met en place des débats sereins et constructifs facilitant l'émergence de réponses collectives : instauration préalable de règles de débat, gestion efficiente du temps, médiation, recherche de l'intérêt général et respect de la parole de chacun ».

Pour le P.A.D.D. (plan d'aménagement et de développement durable), document de travail préalable au futur P.L.U. (plan local d'urbanisme), quatre axes sont évoqués par les membres du cabinet avec d'abord, un cadre de vie à préserver, puis une dynamique de proximité à valoriser. Il faudrait atténuer la coupure entre les Joncs Marins et le centre-ville et renforcer la mixité sociale et générationnelle, ensuite des atouts perruxiens à développer, enfin, des aménagements à réaliser en faveur de la ville durable. Un urbanisme responsable veut favoriser les transports alternatifs à la voiture et limiter l'exposition aux risques naturels et nuisances.

Selon l'édile, «Les contraintes juridico-politiques pesant sur la Petite Couronne montrent que le plan d'occupation des sols (P.O.S.) réalisé en 1974 a été un succès. En novembre 2013, la ville lance la procédure de P.L.U. Mais avec la loi A.L.U.R. de 2013, ouvrant à la création de nouveaux logements sociaux, les communes perdraient l'urbanisme et la compétence intercommunale ». La pensée du Grand Paris avec la Métropole, affirme qu'on ne construit pas assez en petite couronne. Il faudrait doubler le nombre de logements, là où du tissu pavillonnaire existe. «On peut accepter une intercommunalité de proximité, mais on doit garder le pouvoir commu- 
nal d'urbanisme». Or où en est-on? Le gouvernement a accepté de revoir la loi M.A.P.T.A.M. du 27 janvier 2014 en septembre-novembre 2014, en réécrivant l'article 12. La métropole n'est plus compétente pour le P.L.U. mais pour le S.C.O.T. selon le principe des poupées gigognes. Les P.L.U. deviennent intercommunaux. Du point de vue politique : «Nogent et le Perreux-sur-Marne sont depuis toujours sur les termes d'une planification locale. Mais ici, il s'agit de redécoupages intercommunaux de plus de 300000 habitants ». Pour les communes de l'Est parisien, la solution serait de s'appuyer sur une association d'édiles, l'A.C.T.E.P. qui dépasse déjà les 300000 habitants. Il faudrait faire passer au Parlement cette idée : "À l'intérieur du territoire, on garde les P.L.U. au niveau intercommunal. Mais cela s'impose au $1^{e r}$ janvier $2016 »$.

Entretemps et à l'initiative de l'État, une enquête publique est ouverte sur les objectifs du contrat de développement territorial (C.D.T.) Paris-Est, entre Marne et Bois, du 4 mai au 19 juin 2015. Ce contrat, présenté comme outil du Grand Paris, regroupe 6 communes réparties sur deux départements.

Il s'est fixé 14 objectifs à l'issue d'études menées avec les services de l'État et les collectivités territoriales : une étude urbaine réalisée par le Cabinet Seura, puis une étude environnementale par le cabinet Anteagroup. Pour l'Est parisien, il doit émerger un pôle économique, de formation et d'innovation consacré au développement durable, à la vie urbaine et aux grands services urbains (transports, gestion de l'eau....) sur ce territoire.

« Mettre en cuvre une mobilité durable à toutes les échelles, impulser des dynamiques économiques innovantes et durables au bénéfice des populations de l'Est parisien, conforter l'attractivité résidentielle de Paris-Est entre Marne et Bois et faire du territoire une vitrine de la ville durable » s'impose (cf. Le Perreux, notre cité n446, mai 2015, p. 123).

Nous avons décrit précédemment les missions et modes d'intervention du cabinet présentés sur un mode de communication et «marketing », puis la question de l'identité patrimoniale du Perreux. Avec le récit de l'édile, interprétons maintenant le rapport entre les réponses du cabinet à la commande politique et la position du député maire.

\section{La geste urbanistique du député maire lors de l'animation de réunions publiques au Perreux}

L'analyse des réunions publiques va nous permettre de cerner les dimensions symboliques du récit techno-politique du député maire, au contact de la « doxa » des habitants. Ce récit « techno-politique », fondé en bonne part, sur une « imagerie » technique, aurait une usure rapide (L. Sfez, 1993, 2002). Le contexte d'une politique éclatée où les signes ne font plus symboles serait confirmé. Une institutionnalisation dans la durée n'est plus possible. Les conditions qui permettent de voir se développer une réelle démocratie locale et un investissement dans un projet de ville future pour les habitants n'existent pas.

L'hypothèse à discuter est alors la suivante : les réunions publiques perruxiennes ne permettraient pas, pour le moment, de vision globale du projet de ville alors qu'elles attirent beaucoup de gens. Sont-elles représentatifs de l'ensemble des per- 
ruxiens ? Quel projet de territoire pourrait être retenu selon une version « bottom up »?

L'organisation des conférences-débats se fonde sur un temps de programmation et de validation municipale qui ne permet pas une véritable construction d'un sens commun solide avec les habitants. La procédure de concertation par réunions publiques nous paraît trop directive. A contrario, cela supposerait une critique préalablement informée sur les enjeux d'une organisation spatiale métropolitaine. D'une part, les aspects techniques (gares importantes, réseaux techniques de transports, dynamique économique, etc...) enferment tout projet de ville à l'avance dans des exigences de taille. Or, paradoxalement, c'est moins l'intérêt général d'une défense du territoire et du cadre de vie pour l'avenir que l'intégrité de l'espace de vie immédiat qui est évoquée par les perruxiens et relayée par l'édile, lors de la redéfinition du plan local d'urbanisme (P.L.U.). D'autre part, aucun effort de pédagogie, préalable à un débat, n'est vraiment fait localement hormis un affichage assez technique des projets au centre des bords de Marne, à la bibliothèque et sur internet avec une information générale à l'aide de cartes. Un processus de questionnaire internet est proposé aux habitants, en février 2015, sur le site du Perreux. Un accent est mis sur les aspects juridiques de protection du code de l'urbanisme par les urbanistes-animatrices du cabinet, surtout à partir d'octobre. Par exemple, nous notons que deux contraintes seraient à intégrer : d'abord sur le logement (constructions nouvelles plus nombreuses). Une négociation est engagée avec l'État. Ensuite, la contrainte du logement social. D'ici 2025 le seuil proposé à 220 logements/an, semble hors de portée. Il vise la diversité de l'habitat plutôt que la mixité sociale. "Le Perreux ne doit pas devenir une ville de familles bourgeoises ». 9\% de logements sociaux en $1992 ; 15 \%$ aujourd'hui ; 20\% d'ici 2016 : «Il ne faut pas que le passant passe et se rende compte que ce sont des logements sociaux ». La qualité des prestations permettrait de ne pas tomber dans une position « jusqu'auboutiste », peu propice à la conservation de marges de manœuvre. En effet, le préfet peut prendre des arrêtés si le seuil défini de construction n'est pas respecté. Si le délai de carence est dépassé, il impose amende et contrainte. Mais «le seuil de $25 \%$ est impossible à tenir $»$.

Une autre dimension à respecter est l'identité patrimoniale de la commune. Le Perreux est née dans les années 30. L'édile donne aux habitants cette représentation de la ville : alignement des maisons sur de longues rues droites formant quadrillage et respectant l'harmonie des façades ; petits îlots de verdure, comme des poumons verts. Pour préserver la trame viaire existante, l'accueil de nouvelles populations sera limité, pour garder une échelle humaine avec 33000 habitants. Sinon "Il y aura un problème d'écoles, d'équipements et d'impôts. (...) Durant la dernière décennie une certaine densification sur les grands axes a été acceptée. Il faut la poursuivre. On garde alors, autant que possible, l'habitat pavillonnaire à proximité. Il n'y a pas d'expropriation depuis 1978 et l'on acquiert, au fur et à mesure, des terrains sur le centre-ville ». Ainsi, pourrait être poursuivie la densification urbaine du centre-ville, avec $20 \%$ de logements sociaux. L'édile pose une question au Cabinet Citadia, après l'avoir adressée à l'assistance : « le P.L.U. n'organise-t-il pas des règles plus molles de dé- 
fense du cadre de vie, à la différence de l'ancien P.O.S.? » Pour le cabinet, on peut s'appuyer sur des règles strictes de protection de l'environnement en le préservant avec des zonages (ex : en cœur d'îlots, on préserve 10\% d'emprise au sol). Pour l'édile : «il faut y aller en finesse, mais il faut des intentions claires et des règles qui les traduisent $»$. Défendre la hauteur d'habitation (pas plus de quatre étages pour l'ensemble du centre-ville) et la densification sur les grands axes sont une priorité. Cent-cinquante logements par an constituent la limite acceptable. Construire et rappeler cette position défensive, avec l'aide des dispositions du code de l'urbanisme, sera la source principale de légitimité du pouvoir. La longueur des bâtiments telle qu'elle sera perçue par les habitants n'entrera pas en ligne de compte en ce qui concerne les autorisations municipales.

Comme conseil en développement territorial et habitant, je pense qu'il faudrait un certain recul, acquis à l'aide d'une pédagogie plus ouverte sur les représentations, elles-mêmes accompagnant les pratiques urbaines des habitants. Il s'agit là du « perçu-vécu-conçu » de «l'espace des représentations » (H. Lefevbre, 1974). Un projet d'ateliers n'a pas été retenu par la mairie. Un tel travail de débat ouvert sur l'expression d'habitants et leurs représentations semble impossible, vu le « timing » des phases de validation. De plus, ce travail très programmatique, apparaît difficile à rendre lisible, étant donné le contexte complexe. Il s'agirait de plusieurs niveaux d'intervention de composition urbaine pour les gares du Grand Paris Express ${ }^{3}$. Par ailleurs, le récit nous donne une projection close sur un présent ancré dans le passé, et peu de projection pour cet avenir à $10 \mathrm{ou}$ 15 ans. Plus globalement, à l'échelle régionale, l'agenda politique de l'édile le contraint, pour la validation du P.A.D.D. et pour défendre son pouvoir d'urbanisme contre les futurs établissement publics intercommunaux (ils seront « programmés » par l'État en mars 2016), à interpeller les habitants comme si cela faisait leur pain quotidien. Il faut ici avoir en mémoire les éléments de réflexion de l'édile, qui au niveau local, ne peut se départir des interpellations de niveau régional, avec le contexte d'intermédiation des échelles qui se pose à lui. Il y fait référence sous forme d'un compte rendu d'un cheminement décisionnel retranscrit dans la communication du bulletin municipal. En mars 2015, les avis recueillis au cours des réunions et par Internet feront l'objet d'une synthèse par les membres du cabinet, publiée dans le bulletin municipal.

Cette synthèse des avis et orientations générales, validée avec le P.L.U. et attendue notamment, parmi les perruxiens, par ceux qui souhaitent devenir propriétaires en 2015, est proposée au compte-goutte depuis la fin de l'année 2014 et jusqu'à la fin octobre 2015, lors des deux dernières conférences-débats. Elles seront jointes au document d'enquête publique, avec le volet concernant le C.D.T. Entretemps, une validation d'orientations du P.A.D.D. par le conseil municipal du Perreux aura été engagée.

La communication municipale paraît conçue selon l'ingénierie décisionnelle particulière des aléas des retours synthétiques seuls connus du pouvoir. Cela renforce l'idée que le «provisoire » caractérise les stratégies (municipales, administratives). Le provisoire est-il une fatalité qui empêche toute action volontaire et continue ou sert-il au pouvoir local à garder la main sur 
ce qu'il souhaite vraiment faire, au moment qui lui conviendra?

Des contradictions apparaissent avec l'avancée du front d'urbanisation en projet. Mais le discours de l'édile n'en fait pas part. En effet, les orientations du P.A.D.D., mises en avant par le cabinet, paraissent reprises par l'édile pour souligner les traces du passé de la ville depuis les années 30 . Ces traces ont permis de construire une stratégie conservatoire à l'aide d'un parcellaire permettant de préserver ce patrimoine urbain et architectural. Elle aurait bénéficié à tous, préservant bonheur de vivre et cadre de vie de qualité avec les dispositions du code de l'urbanisme.

Pourtant, un début de vision stratégique est tourné vers l'avenir tout en continuant à privilégier une démarche rétrospective à l'aide des projections numériques que sont « power-points », cartes en « $3 \mathrm{D} »$ et « google maps ». Il s'enracine dans l'identité produite par des espaces verts de « la trame verte et bleue ", c'est-à-dire le cadre de vie des bords de Marne qui font l'histoire des guinguettes des années 30 avec le célèbre Parc du Perreux, espace semi-public de splendides maisons de maîtres dont certaines sont classées. L'idée est de mobiliser l'habitant, tout en se référant, de façon subliminale, à l'aménagement de la nouvelle gare de Nogent-Le Perreux sur la ligne $\mathrm{n}^{\circ} 15 \mathrm{du}$ Grand Paris Express quand des questions sont posées, c'est-à-dire rarement.

Une volonté de l'édile de construire un morceau de ville à l'aide des usages sociaux à différentes échelles se manifeste de façon épisodique pour répondre au principe de la constitution commune d'un récit-vision « à 10, 15 années », Mais cela est peu mis en rapport avec des perspectives futures plus globales qui pourraient paraître contradictoires aux échelles supérieures. Pourtant deux autres gares dont l'une impactera un des quartiers du Perreux, celui des Joncs Marins au nord de la ville, sont concernées. Il s'agit des gares du Perreux-Neuilly-Plaisance et de Val-de-Fontenay sur le Grand Paris Express. Enfin, une requalification de 3 entrées de villes est prévue avec l'insertion des nouveaux pôles-gares.

L'application de la loi « N.O.T.R.e. » est prévue pour Janvier 2016 selon les derniers développements qu'impose le préfet de région en juillet 2015. Douze territoires constitueront la future métropole du Grand Paris (M.G.P.) avec les redécoupages sous forme de regroupements intercommunaux nouveaux. Des perspectives plus générales se dessinent au fur et à mesure et cela présente la difficulté d'une mise en débat. Le territoire où le Perreux est pris (l'ancienne « communauté d'agglomération de la Vallée de la Marne ») est envisagé avec 13 communesmembres et 503198 habitants.

Comme composant d'un appareillage technique de grande ampleur, le projet du Grand Paris Express ne peut symboliser l'évidence d'un projet d'avenir pour une société locale. Les conséquences des projets pour l'avenir nous semblent mal perçues et trop lointaines, faute d'activités de délibération. Une communication écrite et cartographique des différentes orientations, phase par phase, paraît insuffisamment vulgarisatrice. Le vocabulaire technique se passe d'un retour réel aux habitants, fruit d'une réflexion construite en commun. Sans communication préalable de l'ensemble des avis recueillis par 
« questionnaire internet » et discussion générale au-delà d'une simple communication par le bulletin municipal sur les retours d'avis, aucun processus d' « empowerment $»^{4} n$ 'est possible. Je m'interroge alors sur la nature et les conditions d'une citoyenneté urbaine sous un prisme plus politique d'une autonomie sociale face à un système territorial que l'on s'approprierait, en dépit de l'accélération des processus métropolitains. Dans un numéro récent de la revue Esprit ${ }^{5}$, les auteurs voient ces processus émergents comme tendant « à réinventer une souveraineté territoriale $»$. Finalement, parler de démocratie dite $« \mathrm{du}$ sommeil » (J. Viard, 2006) et non de démocratie réellement participative, s'expliquerait par « la fausse solution d'un local » (D. Behar, 2015). Des habitants-participants seraient seuls consultés, alors qu'ils (elles) ne s'impliquent pas toujours localement. La démocratie ne représenterait pas la population de passage, mais plutôt celle qui y habite. Le pouvoir local s'illustrerait par une production inadéquate d' " effets de proximité ». Pourtant, critiquer l'étroitesse de l'ancrage du pouvoir local et son manque de représentativité pour un territoire en mutation, véritable boîte noire, ne devrait pas empêcher d'envisager les « transitions énergétiques et médiations politiques » (G. Simondon, 1958, 2012, P. Chabot, 2015). De quoi s'agit-il ? Il s'agit d'appeler à une « transitologie », comme l'ont fait certains auteurs critiques du progrès technique dans l'Amérique du Nord des années 70 (P. Chabot, 2015). L'histoire complexe et la description de ruptures et discontinuités d'états des techniques du passé vont dans ce sens. Avec des transformations d'états et l'abandon historique de types d'économies énergétiques, des bifurcations ont été oubliées. Elles sont peu perceptibles pour un progrès technique asymptotique déterminant les représentations collectives majoritaires. Il s'agit alors de comprendre le « progrès technique » avec ce qui doit être qualifié de « salient revers » ou fronts renversés pour A. Gras, sociologue « passeur » avec le géographe G. Dupuy, des concepts du courant d'historiens des techniques américains avec J. Tarr ou T. Hughes ${ }^{6}$ (A. Gras, 1997).

Pour ces historiens, de nombreuses discontinuités importantes peuvent être perçues comme destructrices d'autres solutions possibles et être associées à des grappes d'innovations techniques. Des effets de ruptures sur un front des innovations techniques structurent différentes tendances évolutives non convergentes. Mais peu à peu, elles sont intégrées avec l'évolution des « large technical systems » ou « macro-systèmes techniques » bouclés sur eux-mêmes dans la phase actuelle. Ce sont alors des « commutateurs de flux d'énergies et d'informations locaux» (J.M.Offner, 1996). Ces « networks of power» complètent les réseaux territoriaux et se rendent indispensables tout en restant invisibles pour une vie socio-économique mondialisée (le modèle étant l'émergence historique du système de l'électricité aux États-Unis).

Finalement, une genèse socio-historique des inventions techniques avec ces moments critiques et leur part d'arbitraire met en perspective les choix aléatoires d'une société donnée, pour une économie énergétique particulière (de bons exemples français sont le choix gouvernemental du moteur diesel automobile ou l'abandon du minitel des années $80 \ldots)$.

Avec cette perspective finale, notre cas d'espèce permettrait d'envisager des scénarii prospectifs 
avec une « visualisation » et une réflexion critique d'une société locale. Il s'agirait d'un système territorial perruxien avec le rapport dialectique entre facteurs d'invariance et de changement. Il permet la reproduction d'un code patrimonial (Y. Barel, 1981). Il faut alors montrer l'importance de ces passages d'états en les figurant comme des expériences de choix politiques à un moment donné.

Par ailleurs, une phénoménologie herméneutique permettra d'évaluer les conditions du mode d'interpellation des habitants et acteurs au Perreux. Un versant est tourné vers la conservation du cadre de vie, et un autre vers ce que l'on peut nommer « modernité technique universelle » (M. Marié, 1989). L'herméneutique prendra place ici, avec la construction d'un récit. Il est la part subjective dans le champ social d'une construction collective. Un exercice biographique se dessine alors (F. Ferraroti, 1983, P. Ricœur, 1990). La dialectique entre structure du récit (explication) et compréhension du monde renverra aux modalités existentielles d'une expérimentation tournée vers l'altérité (P. Ricœur, 1990).

\section{Conclusion}

On ne peut conclure en évaluant l'efficacité symbolique de la proposition faite par l'édile aux perruxiens. Elle est en phase de composition en la forme d'une « représentation sociale, soit une forme de connaissance socialement élaborée et partagée, ayant une visée pratique et concourant à la construction d'une réalité commune » (D. Jodelet, 1993), ou encore comme «création sociale et/ou individuelle d'un schéma pertinent du réel » (J.P. Guérin, H. Gumuchian, 1985). Elle prendra, ou non, dans sa forme signifiante contradictoire. Discuter, avec le récit de l'édile, l'hypothèse d'une difficulté à voir naître les conditions d'une participation démocratique métropolitaine est essentiel. De courtes échéances proposées pour la concertation et la validation du processus décisionnel seraient, à priori, les causes. Mais plus généralement, remarquer aujourd'hui, une absence de critique philosophique de la technique, avec l'exemple de la politique symbolique d'un édile, nous aiderait à poursuivre un questionnement toujours aussi nécessaire (cf. dans cette perspective, les travaux exemplaires de P.Musso, 1997, 2003). Ce questionnement porterait alors sur les rationalités locales développées par un projet de Grand Paris qui ne compenserait pas le caractère éclaté et fragmentaire d'une totalité non articulée dans ses composantes techniques sectorielles ( « totalité fragmentaire et fragmentée » d'une technique planétaire, selon le philosophe K. Axelos dans son ouvrage de 2010).

Ouvrons la réflexion sur l'intérêt d'un récit d'un « progrès subtil », dosé en fonction des moyens techniques et d'états de transition. L'opposer dialectiquement, à un « progrès utile » (P. Chabot, 2015), « multilinéaire et par capitalisation », parfaitement associable à un imaginaire de la croissance urbaine, nous sortirait d'une fascination pour la technique. L'incompréhension des facteurs génétiques qui la déterminent disparaîtrait avec l'explication des transitions énergétiques, par exemple (G. Simondon, 1958, 2012, P. Chabot, 2015). Des «minorités actives » (S. Moscovici, 1972), comme le courant des écologistes « verts », seraient suffisamment critiques des évolutions techniques prométhéennes pour arriver à s'emparer du récit proposé par l'édile et construire leur propre rapport au monde. Elles 
construiraient leur propre vision du monde, afin de nous engager vers une limitation des mobilités urbaines et de leurs effets environnementaux, l'ensemble constituant un nouvel élan de vie démocratique.

\section{$R \cdot E ́ E \cdot E \cdot R \cdot E \cdot N \cdot C \cdot E \cdot S$}

AXELOS (K), Ce qui advient. Fragments d'une approche, 2009, Encre Marine - En quête de l'impensé, 2010, Encre marine, Paris.

ARDOINO (J), «Complexité », in L. Sfez et alii, Dictionnaire critique de la communication, T.1, 1993, P.U.F., Paris, pp. 196-198.

BAREL (Y), « Modernité, code, territoire », 1981, $\mathrm{n}^{\circ}$ 10-11, Annales de la recherche urbaine, Paris. BAUBY (P), L'État-stratège. Le retour de l'État. 1991, Éditions ouvrières, Paris.

BLONDIAUX (L), Le nouvel esprit de la démocratie. Actualité de la démocratie participative, Collection la République des idées, 2008, Seuil, Paris.

CHABOT (P), L'Âge des transitions, 2015, P.U.F., Paris.

CHOAY (F), L'Urbanisme, utopies et réalités. Une anthologie, 1966, Points-Seuil, Paris. Présentation de Webber Melvin M., L'Urbain sans lieu ni bornes, La Tour-d'Aigues, France, éd. de l'Aube, 1996, 123 p.

DAVEZIES (L), PECH (T), « La nouvelle question territoriale», 3.09. 2014, Terra Nova, Paris. DUPUY (G), L'urbanisme des réseaux. Théories et méthodes, 1991, Armand Colin, Paris.

DUBOIS-TAINE (G) et CHALAS (Y), La ville émergente, 1997, Ed. de l'Aube, Paris.

DONZELOT (J), ESTEBE (P), L'État-animateur. Essai sur la politique de la ville, 1994, Esprit, Paris. ÉCOLE D'URBANISME DE PARIS, Sixième édition des Journées Grand Paris, 19 et 20 Mars 2015, Marne-La-Vallée.

ELLUL (J), « Les 'idées-images' de la ville de l'homme quelconque », in L'idée de la ville, 
actes du colloque international de Lyon, Éditions du Champ Vallon, Seyssel, 1984 (pp. 37-43). Penser la ville. Choix de textes philosophiques. ANSAY(P.) et SCHOONBRODT(R), 1989,AAM Éditions, p. 315.

ESPRIT, L'avenir du pouvoir local, $\mathrm{n}^{\circ} 412$, février 2015, Paris.

FERRAROTI (F), Histoire et histoires de vie. La méthode biographique dans les sciences sociales, 1983, Librairie des Méridiens, Paris.

LACOSTE (Y), Dictionnaire de géopolitique, 1995, P.U.F., Paris.

GAUDIN (J.P.), L'aménagement de la société, 1979, Anthropos, Paris. Technopolis, 1985, P.U.F., Paris.

GRAS (A), Les macro-systèmes techniques, 1997, Que-sais je ? P.U.F., Paris.

GILLI (F), Grand Paris. L'émergence d'une métropole, 2014, P.F.S.P., Paris.

JOUVE (B), « Politiques publiques et empowerment. L'exception française », Économie et humanisme, n³79, 2006, pp. 99-101.

JODELET (D), Les représentations sociales, 1993, P.U.F., Paris.

LEFEVBRE (H), Production de l'espace, 1974, Anthropos, Paris.

MARIE (M.), « Penser son territoire. Pour une épistémologie de l'espace local », in Encyclopédie Diderot, dirigée par R. Brunet et F. Auriac, novembre 1987.

MORVAN (B), « De l'utopie à l'utopisme : le cas de l'urbanisme des réseaux », n²8, Quaderni, Hiver 1995-1996. « Représentations de la métropolisation. Nouveaux lieux, nouveaux territoires », n॰83, Quaderni, Hiver 2013-2014. « Émergence d'un récit contradictoire du périurbain dans le Grand Paris : le cas de l'Institut du Mont Ezard à Villecresnes dans le sud-est du Val-de-
Marne », n86, Quaderni, Hiver 2014-2015. MOSCOVICI (S), De la nature : pour penser l'écologie, 2002, Métaillié, Paris. "Pourquoi les écologistes font-ils de la politique ? », 1978 in S. Moscovici et R. Dumont. Volume $n^{\circ} 49$, Combats, Seuil, Paris.

MUSSO (P), Critique des réseaux, 2003, P.U.F., Paris.

PANERAI(P), Paris métropole. Formes etéchelles $d u$ Grand Paris, 2008, Éd. La Villette, Paris. PARROCHIA (D), Philosophie des réseaux, 1993, P.U.F., Paris.

PUMAIN (D), OFFNER (J.M) (directeurs), Réseaux territoriaux. Significations croisées, 1998, Éditions de l'Aube, Armand Colin, Paris. OFFNER (J.M.), « 'Réseaux' et 'Large Technical System' : concepts complémentaires ou concurrents ? » in Flux, n²6, 1996. pp. 17-30.

RAFFESTIN (C), Pour une géographie du pouvoir, 1980, Litec, Paris.

RICOEUR (P), Du texte à l'action. Essais d'herméneutique II, 1985, Seuil, Paris. Soi-même comme un autre, 1990, 1997, Points-Seuil, Paris. SIMONDON (G), Du mode d'existence des objets techniques, 1958, Aubier, réédition. 2012, Flammarion, Paris.

SFEZ (L), La politique symbolique, 1993, P.U.F., Quadrige, Paris. Technique et idéologie. Un enjeu de pouvoir, 2002, P.U.F., Paris.

HUGHES (T), Networks of power : Electrification in Western Society, 1880-1930. Baltimore: Johns Hopkins University Press, 1983.

VIARD (J), Éloge de la mobilité, 2006, La Tourd'Aigues, Éditions de l'Aube, Paris.

VELTZ (P), Mondialisation, villes et territoires : l'économie en archipel, 1996, P.U.F., Paris.

WEYEMBERG (M), Aspects de l'utopisme contemporain, 1991, Vrin, Paris. 


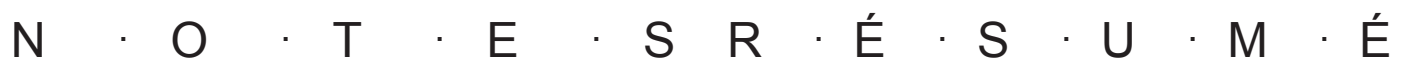

1. Table ronde des matins du C.G.M.E.D.D. (conseil général du ministère de l'Écologie et du Développement durable) du 5.02.2015: « Métropoles et territoires » avec P. Veltz, Chercheur et directeur général de l'établissement public de Saclay, J. Roland, maire de Nantes, et D. Läppel, professeur à l'université Hafen-City de Hambourg.

2. D. Mangin, Paris-Babel. Une mégapole européenne, Éditions Descartes et Cie, 2013. Y sont mentionnés en introduction les travaux de l'équipe Descartes, à partir de 2009-2010, selon un contexte de mobilité. Ils se distinguent de ceux de R. Castro, $\mathrm{S}$. Denisoff et F. Léonhart où l'on insiste autrement sur des centralités symboliques à concevoir en banlieue (notamment la partie nord avec le projet de nouveaux équipements publics importants).

3. J.M. Offner, Intervention de l'urbaniste et politologue au forum de Libération « À nous le Grand Paris » au 104 à Paris, le 26 novembre 2014, Atelier : « Un métro nommé futur ».

4. B. Jouve, « Politiques publiques et empowerment. L'exception française », Économie et humanisme, n³79, 2006, pp. 99-101. L'économiste Amartya Sen parle de « capacitations ».

5. Esprit, L'avenir du pouvoir local, ${ }^{\circ} 412$, Articles de B. Giblin: «L'obsession du local : une exception française? »p. 64, D. Behar : «Changer les institutions ou changer les pratiques? », p. 85 et M. Vanier : « Démocratie locale : changement d'horizon?», p. 109 et entretien : «Y a-t-il une bonne échelle locale? » entre D. Behar et J. Lévy, p. 109, Paris, Février 2015. 6. T. Hughes, Networks of power : Electrification in Western Society, 1880-1930. Baltimore: Johns Hopkins University Press, 1983.
La réforme territoriale du 27 janvier 2014 aiderait à questionner, à nouveau, le sens de l'interpellation du pouvoir d'État et de la capitale, Paris, au Perreuxsur-Marne. Les communes périphériques de première couronne de la région parisienne devront définir leur avenir avec un plan local d'urbanisme. Elles devront composer, à 1'horizon 2016, avec des nouvelles orientations métropolitaines. De nouvelles unités urbaines et des redécoupages territoriaux intercommunaux de plus grande ampleur seront les éléments envisagés. Nous faisons l'hypothèse qu'un questionnement radical, ouvert au jeu du monde (K. Axelos, 1979, 1991) doit être conservé dans toute la tension qu'il fait naître. Nous poursuivons ainsi, notre interrogation sur le sens d'une praxis des aménageurs et édiles accompagnant la phase cruciale mais incertaine, d'institutionnalisation des métropoles de janvier 2014 (B. Morvan, 2014, 2015). Cette perspective permettrait de s'ouvrir au récit urbain de l'édile. Notre orientation serait donc tournée vers l'appréciation d'un récit à portée symbolique pour compenser le contexte de fragmentation spatiale d'une totalité urbaine. Cette totalité ne nous paraît pas encore définie de façon stable (B. Morvan, 2014). Les réunions publiques organisées au Perreux-sur-Marne, pour l'instruction du P.A.D.D. (plan d'aménagement et de développement durable), permettraient d'identifier les éléments symboliques d'un récit à portée collective. Des "minorités actives 》 (S.Moscovici, 1972, 1978, 2002), suffisamment critiques des évolutions techniques prométhéennes, s'empareraient ou non du récit proposé par l'édile pour construire leur propre vision du monde. L'avenir incertain, de façon plus marquée qu'à l'époque de l'émergence du mouvement écologiste des années 70, 
ouvrirait à l'appel du « centre énigmatique du jeu du monde » où se situe l'« impensé» de nature poétique (K. Axelos, 2009, 2010). La limite incertaine d'une forme métropolitaine, avec des termes opposés et non conciliables, ouvre à ce qui manque : l'impensé d'une enquête infinie. Une philosophie critique de la technique et l'avenir d'une démocratie participative sont les termes futurs d'une telle enquête.

\section{Abstract}

The territorial reform of January 27. 2014 would help to question, once again, the meaning of the intervention of State authority and that of the capital, Paris, concerning le Perreux-sur-Marne. The municipalities in the first peripheral ring of the Paris region will have to define their future with a local urbanism plan. Beginning 2016, they will have to compose with new metropolitan orientations. New urban units and inter-territorial redistribution on a larger scale will be envisaged. We assume that a radical questioning opened on « the game world» (K. Axelos 1979, 1991) should be maintained in all the tension it engenders. We thus pursue our interrogation about the sense of a praxis of developers and aediles accompanying the crucial but uncertain phase of institutionalizing the metropolitan areas of January 27. 2014 (B. Morvan, 2014, 2015). This would allow us to unfold the aedile's urban narrative. Our focus would be directed towards the appreciation of a symbolic narrative to compensate the context of spatial fragmentation of urban totality. All this does not seem as yet stably defined. Public meetings for le Perreux-sur-Marne's P.A.D.D. (planning programming sustainable development) would identify the symbolic elements of a story with a collective scope. «Active minorities » (S. Moscovici, 1980), critical enough of Promethean technical developments, would seize or not the narrative proposed by the Deputy-Mayor to build their own vision of the world. The uncertain future, more marked than at the time of the emergence of the environmental movement of the 70s, would open to the call of the « enigmatic center of the game world». It is the place where exist « the unthought of » poetic nature (K.Axelos, 2009, 2010). The uncertain limit of a metropolitan form, with opposite and irreconcilable terms, opens what is missing: the unthought of infinite survey. A critical thought of the technique and the future of a participatory democracy are the terms of such a survey. 
